\title{
The Deceptive Mushroom: Accidental Amanita muscaria Poisoning
}

\author{
Francesca Irene Rampolli ${ }^{1}$, Premila Kamler ${ }^{1}$, Claudio Carnevale Carlino ${ }^{1}$, Francesca Bedussi ${ }^{2}$ \\ ${ }^{1}$ Department of Internal Medicine, Ospedale Regionale di Lugano, Switzerland \\ ${ }^{2}$ Division of Clinical Pharmacology and Toxicology, Institute of Pharmacological Sciences of Southern Switzerland, Ospedale Regionale di Lugano,
} Switzerland

Received: $14 / 12 / 2020$

Accepted: 08/01/2021

Published: 02/02/2021

How to cite this article: Rampolli FI, Kamler P, Carnevale Carlino C, Bedussi F. The deceptive mushroom: accidental Amanita muscaria poisoning. EJCRIM 2021;8: doi:10.12890/2021_002212.

Conflicts of Interests: The Authors declare that there are no competing interests.

This article is licensed under a Commons Attribution Non-Commercial 4.0 License

\section{ABSTRACT}

Amanita muscaria is considered to be one of the most remarkable and beautiful mushrooms. It has a red or orange cap covered with small white plaques and its distinctive appearance makes accidental and severe intoxication very rare. Its consumption is sometimes used as a means of suicide or it can be consumed for its psychedelic effects, and in some cases, it can be mistaken for edible species. In this paper, we will discuss a patient who fell into a coma after accidental Amanita muscaria poisoning. Rapid identification of the mushroom allowed the regression of symptoms and discharge from the hospital on the fourth day after consumption.

\section{LEARNING POINTS}

- Amanita muscaria is one of the most remarkable mushrooms for its distinctive appearance, but sometimes it can be mistaken for edible species.

- Amanita muscaria is a highly poisonous mushroom; the primary effects usually involve the central nervous system, and in severe poisoning, symptoms may manifest with coma and in rare cases lead to death.

- The rapid and correct identification of this mushroom is important for optimal risk assessment and in order to prescribe the best therapy.

\section{KEYWORDS}

Amanita muscaria, poisoning, ibotenic acid, muscimol, coma

\section{INTRODUCTION}

Amanita muscaria (fly agaric) is commonly seen throughout continental Europe and the UK from July to October. This mushroom has a red or orange cap with small white plaques. Unintentional poisoning is rare because of its distinctive appearance and its known toxic peculiarities. In recent years, the consumption of the red fly agaric has increased among young people due to its hallucinogenic properties. Although the prevalence of its consumption is lower than that for marijuana and hashish, it is presumed that in the future there will be more patients poisoned by hallucinogenic mushrooms.

It may sometimes be mistaken for the edible species Amanita caesarea. The clinical manifestation related to ingestion is characterized by central nervous system (CNS) symptoms such as confusion, dizziness, tiredness and visual and auditory perceptual changes. Gastroenteric symptoms are uncommon. In extremely severe poisoning, there can be coma, and in rare cases, circulatory and respiratory failure leading to death ${ }^{[1]}$.

This is a case of fly agaric poisoning in a healthy 44-year-old man, who was promptly treated with gastric lavage, activated charcoal and symptomatic treatment. After 4 days he was discharged without further consequences. 


\section{CASE DESCRIPTION}

A 44-year-old-patient, with no previous medical history, was admitted to the emergency department (ED) for malaise, nausea and altered consciousness. His heart rate was $70 \mathrm{bpm}$, tympanic temperature was $35.8^{\circ} \mathrm{C}$, pulse was $65 \mathrm{bpm}$, supine blood pressure was $165 / 95$ and $\mathrm{SpO} 2$ was $91 \%$ on room air. Several minutes after admission, the patient became unconscious, not responding to verbal stimuli nor physical stimuli. According to his wife, an hour prior to hospitalization he had ingested approximately half a kilo of mushrooms that he had collected himself. His wife showed pictures of the mushrooms ingested, which were sent to the mycologist, who identified them as Amanita muscaria (Fig. 1). The poison advice centre was then immediately contacted, which advised us on how to manage the patient.
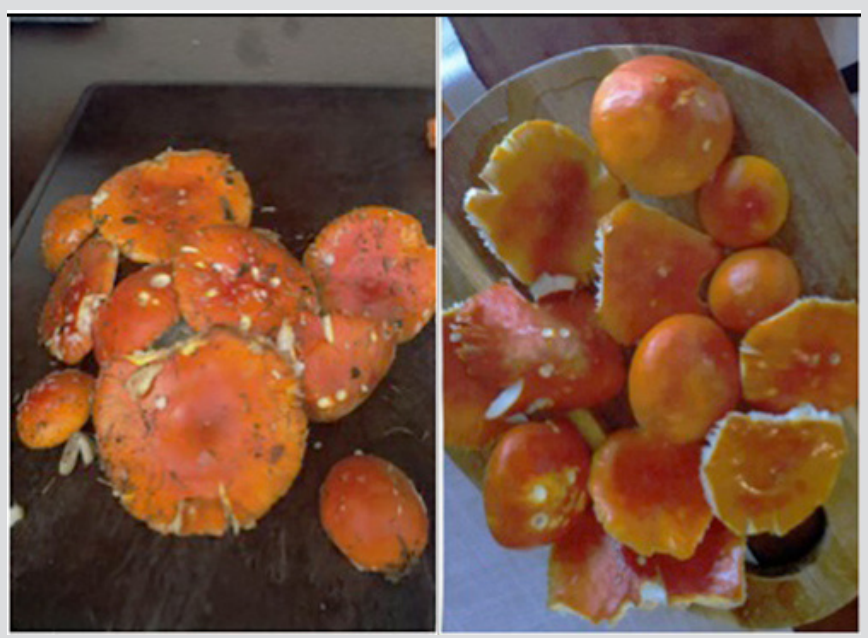

Figure 1. The mushrooms collected by the patient, and after washing them. White plaques were lost and the mushrooms looked like Amanita caesarea

In the meantime, the patient's condition worsened with a GCS score of 4, with normal vital parameters.

After endotracheal intubation, gastric lavage was performed, achieving the elimination of fragments of mushroom from the stomach. ECG monitoring showed no changes in rhythm. Serum creatinine, urea and liver enzymes were normal. Analytical toxicology assessment excluded other causes of poisoning, in particular by psychotropic drugs or alcohol. The patient received activated charcoal ( $1 \mathrm{~g} / \mathrm{kg}$ ) and fluid intravenously. He was then transferred to the ICU. On admission to the ICU, the patient's condition remained serious, and intensive salivary secretion and increased secretion in the airways were observed.

After 72 hours the patient's condition improved, and we observed the appearance of responses to external stimuli and decreased secretion of saliva. The patient was extubated. On the fourth day he was discharged from the hospital.

\section{DISCUSSION}

Amanita muscaria is one of the most recognizable and widely encountered mushrooms in popular culture. Despite its easily distinguishable features, cases of accidental ingestion do exist.

During heavy rain, its characteristic white plaques may wash off and Amanita muscaria can appear similar to - and be mistaken for - Amanita caesarea, an edible mushroom (Fig. 1). Brvar et al. reports that in Slovenia, $90 \%$ of cases of mushroom poisoning are due to the accidental ingestion of Amanita muscaria while being mistaken for Amanita caesarea ${ }^{[2]}$.

Amanita muscaria is a highly poisonous mushroom due to the fact that it contains psychoactive alkaloids: muscarine, ibotenic acid and muscimol. The latter two substances are structurally similar to gamma-aminobutyric acid (GABA) and they act as neurotransmitters in the CNS, stimulating glutamate receptors ${ }^{[3]}$. After mushroom ingestion, ibotenic acid and muscimol are rapidly absorbed by the gastrointestinal tract and cross the blood-brain barrier via an active transport system [4]. Ibotenic acid is rapidly and spontaneously decarboxylated to muscimol, which appears to be the agent responsible for the majority of symptoms, although muscimol may be actively converted to ibotenic acid via glutamate decarboxylase. The alternation between muscimol and ibotenic acid is responsible for the fluctuation in CNS symptoms ${ }^{[3]}$. Symptoms start 30 minutes to 2 hours after ingestion and the primary effects usually involve CNS manifestations, often alternating between stimulation/excitation and depression. They begin as a state of confusion, dizziness, agitation, ataxia, visual and auditory perceptual changes, space distortion and a lack of awareness of time ${ }^{[3]}$. Nausea, vomiting and diarrhoea are uncommon ${ }^{[3]}$. Tachycardia, bradycardia and hypertension may also occur. Both hypo- and hyperthermia have been reported. Respiratory depression and bronchorrhoea are rare. 
Mydriasis, miosis and metabolic acidosis are also possible. In severe poisoning, symptoms may manifest with coma and in rare cases lead to death ${ }^{[1]}$. A famous case is that of Count Achilles de Vecchi, an Italian diplomat residing in Washington, who died in 1897 after ingesting a large amount of mushrooms.

The average duration of clinical manifestation is $8-24$ hours ${ }^{[5]}$; only 1 case described a 5 -day paranoid psychosis ${ }^{[2]}$. In our report, the state of coma lasted 72 hours, and to our knowledge, it represents the first described case of prolonged coma. We believe that it was due to the quantity of ingested mushrooms (13 mushroom caps) and that the rapid treatment applied was decisive for the good prognosis.

The treatment of patients poisoned with red fly agaric should be conducted in hospital. Gastric lavage and symptomatic treatment should be carried out as soon as possible. If necessary, restraint or sedation should be employed for agitated and/or delirious patients. Benzodiazepines are generally effective in controlling agitation, combativeness and muscular overactivity, but may result in respiratory depression. In the case of coma or lack of protective reflexes, intubation and ventilation may be required. ECG, fluid and electrolyte balances should be monitored. There is no antidote and as symptoms are both cholinergic and anticholinergic, atropine and physostigmine are contraindicated.

In conclusion, the rapid and correct identification of the mushroom is imperative (when possible) for optimal risk assessment, and it is mandatory in order to prescribe the best therapy. Clinical manifestations of Amanita muscaria poisoning are variable, but the mortality depends on the amount of mushroom ingested and on symptomatic/supportive therapy management. In symptomatic patients, general supportive measures and decontamination should be undertaken as soon as possible. A delay in gastric lavage can prolong the absorption time and increase the amount of poison absorbed.

\section{REFERENCES}

1. Satora L, Pach D, Butryn B, Hydzik P, Balicka-Slusarczyk B. Fly agaric (Amanita muscaria) poisoning, case report and review. Toxicon 2005;45:941-943.

2. Brvar M, Mozina M, Bunc M. Prolonged psychosis after Amanita muscaria ingestion. Wien Klin Wochenschr 2006;118:294-297.

3. Michelot D, Melendez-Howell LM. Amanita muscaria: chemistry, biology, toxicology, and ethnomycology. Mycol Res 2003;107(Pt 2):131-146.

4. Mikaszewska-Sokolewicz MA, Pankowska S, Janiak M, Pruszczyk P, Łazowski T, Jankowski K. Coma in the course of severe poisoning after consumption of red fly agaric (Amanita muscaria). Acta Biochim Pol 2016;63:181-182.

5. Vendramin A, Brvar M. Amanita muscaria and Amanita pantherina poisoning: two syndromes. Toxicon 2014;90:269-272. 